Martínez-Fernández, D. (2021). Comportamiento diurno de cetáceos en el Área de Conservación Guanacaste, Costa Rica. Revista de Biología Tropical, 69(Suppl. 2), S304-S312. https://doi.org/10.15517/rbt.v69iS2.48745

\title{
Biología Tropical
}

https://doi.org/10.15517/rbt.v69iS2.48745

\section{Comportamiento diurno de cetáceos en el Área de Conservación Guanacaste, Costa Rica}

Damián Martínez-Fernández; ; (D) https://orcid.org/0000-0002-8498-7919

1. Director del Departamento de Política y Conservación, Federación Costarricense de Pesca, San José, Costa Rica; damian.martinezcr@gmail.com

Recibido 11-XI-2020. Corregido 9-XII-2020. Aceptado 20-VII-2021.

\section{ABSTRACT}

Cetacean diurnal behavior in the Guanacaste Conservation Area, Costa Rica

\begin{abstract}
Introduction: Three species of cetaceans, the pantropical spotted dolphin (Stenella attenuata attenuata), the bottlenose dolphin (Tursiops truncatus) and the humpback whale (Megaptera novaeangliae), are important components of the marine fauna of the Guanacaste Conservation Area (ACG). However, basic biological information on the behavior and occurrence of these species remains scarce.

Objective: The objective of this study is to determine the diurnal behavior of the three-mentioned species in the ACG.

Methods: The north Pacific of Costa Rica, from Bahía Salinas to the Marine Sector of ACG was sampled between May 2005 and May 2006. The behavior scanning method was used to identify the general behavior of the cetacean groups.

Results: Encounters were recorded for the three species, in a total of 36 sightings, but only for S. attenuata and $M$. novaeangliae for considerable periods of time. Stenella attenuata exhibited a wide variety of behaviors, being foraging $\left(\mathrm{X}^{2}=29.42\right.$; d.f. $\left.=1 ; \mathrm{P}<0.001\right)$ and travelling $\left(\mathrm{X}^{2}=38.33\right.$; d.f. $\left.=1 ; \mathrm{P}<0.001\right)$ significantly higher during the rainy season. Predominant behaviors for the humpback whale were mother-calf association, social and travelling; being the latter higher during the rainy season $\left(X^{2}=78.16\right.$; d.f. $\left.=1 ; P<0.001\right)$.

Conclusions: Behaviors such as foraging and mother-calf were the most representative among species and the most frequent. This suggests that the area can be considered as a critical habitat for these species of cetaceans, with particular importance for highly migratory species such as humpback whales.
\end{abstract}

Key words: dolphins; humpback whales; Stenella attenuata; Megaptera novaeangliae; cetacean behavior; mother-calf association; marine conservation.

El sector marino del Área de Conservación Guanacaste (ACG) posee $430 \mathrm{~km}^{2}$ de extensión en donde se incluyen algunos sitios de relevancia para la biodiversidad marino-costera, como el Archipiélago Islas Murciélago, Playa Nancite, Sitios Ramsar de Potrero Grande y Laguna Respingue (Bassey-Fallas 2010; Fonseca et al.,
2009; Zamora-Trejos \& Cortés, 2009). Dentro de estos sitios se distinguen ecosistemas altamente diversos como los manglares, arrecifes coralinos y lagunas costeras, así como también especies de tortugas marinas y cetáceos (Cortés, 1996; Cortés, 2017; Fonseca et al., 2019; Loría-Naranjo, Samper-Villarreal, \& Cortés, 
2014; Martínez-Fernández, Montero-Cordero, \& May-Collado, 2011; Zamora-Trejos \& Cortés, 2009).

La relevancia biológica dentro del sector marino del ACG y las zonas adyacentes al norte del área ha sido ampliamente abordada, por ejemplo, se han decretado tanto áreas silvestre protegidas (e.g. Parque Nacional Santa Rosa, Área Marina de Manejo Bahía Santa Elena) como también identificado sitios de importancia para la conservación marina (SIC) con base en la representatividad biológica y amenazas asociadas (SINAC, 2008). Algunos de estos SIC identificados son el Golfo de Papagayo, Punta Santa Elena, Bahía Santa Elena y Punta Descartes, y todos tienen como uno de sus elementos focales las áreas de agregación de cetáceos (SINAC, 2008).

$\mathrm{Si}$ bien los cetáceos aparecen como un grupo taxonómico relevante para el ACG, a la fecha solo se sabe que las especies con mayor proporción de avistamientos son delfines manchados pantropicales (Stenella attenuata attenuata), delfines nariz de botella (Tursiops truncatus) y ballenas jorobada (Megaptera novaeangliae) (Martínez-Fernández et al., 2011; Martínez-Fernández, Montero-Cordero, \& Palacios-Alfaro, 2014; May-Collado \& Morales-Ramírez, 2005). Por su abundancia a lo largo del año, los delfines manchados podrían considerarse residentes de las Islas Murciélago, la Península de Santa Elena y Bahía Culebra, en donde los patrones de alimentación parecen estar relacionados con la disponibilidad, distribución y arreglo espacial de las presas (MayCollado \& Morales-Ramírez, 2005). Además, los cambios estacionales en la abundancia relativa de los delfines manchados en estos lugares, parecen variar con la disponibilidad de alimento (May-Collado \& Forcada, 2012). El delfín $T$. truncatus se observa buena parte del año y se agrega en grupos más grandes frente a Bahía Santa Elena (Martínez-Fernández et al., 2014).

En el caso de las ballenas jorobadas, las aguas costeras del país son utilizadas para reproducción y crianza (Rasmussen, Calambokidis, \& Steiger, 2002) durante los meses de diciembre a abril por el segmento de población o unidad poblacional de Centroamérica (en inglés, DPS, Distinct Population Segment) (Calambokidis et al., 2000; Rasmussen, Calambokidis, \& Steiger, 2012; Steiger et al., 1991) y durante los meses de junio a noviembre por el 'stock' G ( Acevedo \& Smultea, 1995; Acevedo et al., 2017; Palacios-Alfaro et al., 2009; Rasmussen et al., 2007). En el Pacífico norte del país las áreas de mayor agregación de grupos competitivos de machos y madres con crías de ballenas jorobadas se observan en las cercanías de la Península de Santa Elena, mientras que ballenas solitarias son más frecuentes entre Golfo de Papagayo y las Islas Murciélago (Martínez-Fernández et al., 2014).

A pesar de estos esfuerzos de investigación en la zona, aún existen vacíos de información sobre la biología, ecología y comportamiento de las especies de cetáceos que ocurren en el área, lo cual limita la toma de decisiones y la generación de estrategias de conservación para el territorio marino costarricense. El objetivo de este estudio es determinar el comportamiento diurno de los cetáceos en el ACG, específicamente desde Bahía Salinas hasta el Archipiélago de las Islas Murciélago.

\section{MATERIALES Y MÉTODOS}

Área de estudio: En el Pacífico norte de Costa Rica, el área de trabajo se delimitó mediante polígono desde Puerto Soley, dentro de Bahía Salinas, hasta el Archipiélago Islas Murciélago en el Sector Marino del ACG, pasando por el Golfo de Santa Elena hasta llegar a la comunidad de Cuajiniquil (Fig. 1). Esta zona presenta la dinámica de las estaciones generales del país y sigue el patrón presentado por Waylen et al. (1996), donde se presenta una época de lluvias entre mayo y noviembre y una época seca entre diciembre y abril, además de estar bajo influencia de un afloramiento costero que coincide con la época seca (Alfaro \& Cortés, 2012; Alfaro \& Cortés, 2021).

Registro de datos: Basados en estudios previos en Isla Murciélago y Bahía Culebra (May-Collado \& Morales-Ramírez, 2005), se 


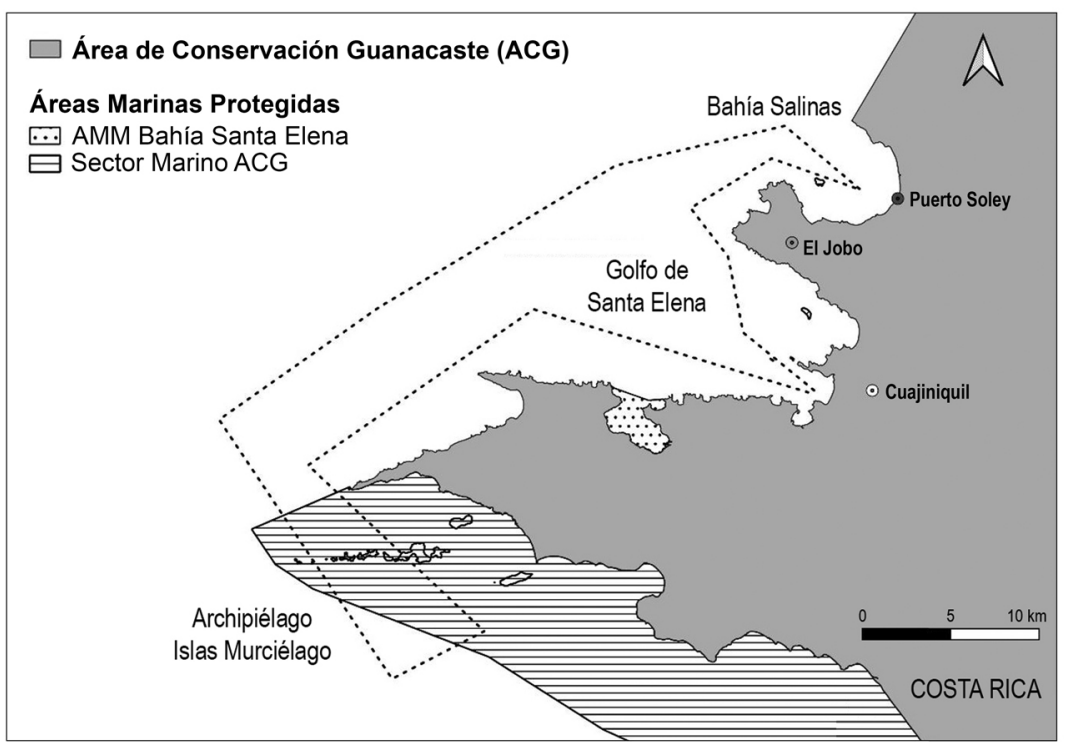

Fig. 1. Recorrido definido para el muestro de cetáceos en el Pacífico norte de Costa Rica, desde Puerto Soley en Bahía Salinas, hasta el Archipiélago de Islas Murciélago en el Sector Marino del Área de Conservación Guanacaste, incluyendo el Golfo de Santa Elena; Área de Conservación Guanacaste.

Fig. 1. Defined route for the sampling of cetaceans in the North Pacific of Costa Rica, from Puerto Soley in Bahía Salinas, to the Archipelago of Murcielago Islands in the Marine Sector of the Guanacaste Conservation Area, including the Gulf of Santa Elena; Guanacaste Conservation Area.

realizaron recorridos entre mayo de 2005 y mayo de 2006, con transectos de banda de $1 \mathrm{~km}$ de ancho para ubicar cetáceos. Los recorridos se realizaron con dos observadores a bordo en botes fuera de borda de fibra de vidrio con motores Yamaha de 75 caballos de fuerza de 2 tiempos. El área de muestreo se estableció de tal forma que permitiera abarcar la mayor área posible, para aumentar la probabilidad de avistamientos. Para esto se siguió una misma ruta de navegación para cada zona de muestreo. Las rutas se recorrieron de tres a cuatro días consecutivos mensualmente, con un promedio de ocho horas de esfuerzo diario, entre las 06:00 y 14:00 hr.

Se utilizó una categorización de comportamientos basándose en otros trabajos (Baird \& Dill, 1995; May-Collado et al., 2005) y adaptado para el presente estudio por tener varias especies de cetáceos involucradas (Tabla 1). En el caso de las ballenas jorobadas, aunque al ser dos poblaciones distintas que llegan al país, como se indicó previamente, ellas utilizan las aguas costeras para reproducción y crianza. De esta manera para este estudio no se segregan los análisis por poblaciones, si no por especie.

Se consideró como un encuentro todos aquellos registros que se realizaron dentro del área de muestreo. A un grupo, como los individuos que tenían actividades coordinadas y estaban cercanos unos a otros (Mann, 2000). El registro se basó en datos de estados del comportamiento, los cuales son patrones de conducta de larga duración (Martin \& Bateson, 2007). Para su medición, se observaron los primeros 5 minutos de la actividad realizada, para saber qué tipo de comportamiento presentaban, el tamaño del grupo, observar con detenimiento a los individuos, determinar sus actividades y tomar fotografías. Seguidamente, durante cada dos minutos se empleó el método de escaneo de comportamiento (Altmann, 1974), para registrar el comportamiento dominante. Cada encuentro finalizó al llegar al límite de 
TABLA 1

Descripción de los estados de comportamiento para cetáceos. Adaptación propia con base en Baird y Dill (1995) y May-Collado y Morales-Ramírez (2005)

TABLA 1

Description of cetacean behavioural states. Adaptated from Baird and Dill (1995) and May-Collado and Morales-Ramírez (2005)

\begin{tabular}{|c|c|}
\hline Categoría & Descripción \\
\hline Forrajeo & $\begin{array}{l}\text { Respiraciones no sincronizadas, sin dirección, misma velocidad, desplazamiento mínimo, } \\
\text { buceos continuos en algunos individuos. Posible comportamiento simbiótico con aves marinas. }\end{array}$ \\
\hline Desplazamiento-forrajeo & $\begin{array}{l}\text { Respiraciones sincronizadas con dirección de desplazamiento constante, misma velocidad, } \\
\text { pausas esporádicas para el buceo. Posible comportamiento simbiótico con aves marinas. }\end{array}$ \\
\hline Alimentación & $\begin{array}{l}\text { Presencia de presas o parte de ellas, será el tiempo desde el primer ataque hasta que la última } \\
\text { pieza de alimento sea consumida. Posible comportamiento simbiótico con aves marinas. }\end{array}$ \\
\hline Descanso & $\begin{array}{l}\text { Respiraciones sincronizadas, dirección del desplazamiento constante muy lenta y sin mucho } \\
\text { movimiento. Puede haber animales flotando en superficie }\end{array}$ \\
\hline Desplazamiento & $\begin{array}{l}\text { Respiraciones sincronizadas, dirección del desplazamiento constante, alta velocidad, } \\
\text { regularmente saltan fuera del agua. Si se observan comportamientos con aves o peces saltando } \\
\text { se considera desplazamiento-forrajeo. }\end{array}$ \\
\hline Socialización total & $\begin{array}{l}\text { Movimientos interactivos entre la totalidad del grupo de animales, no asociados a la captura de } \\
\text { presas. En el caso de ballenas jorobadas eran grupos de machos competitivos. }\end{array}$ \\
\hline Socialización parcial & $\begin{array}{l}\text { Movimientos interactivos entre cierto número de individuos del grupo de animales, no } \\
\text { asociados a la captura de presas. Posible interacción con botes. En el caso de ballenas } \\
\text { jorobadas eran grupos de machos competitivos. }\end{array}$ \\
\hline Madre-cría & Solo en caso de ballenas jorobadas, encuentros en donde se presentaron crías con un adulto. \\
\hline Buceo & $\begin{array}{l}\text { Solo en caso de ballenas jorobadas, los individuos salen a respirar un tiempo corto cerca de } 5 \\
\text { minutos y se sumergen de } 15 \text { a } 20 \text { minutos. }\end{array}$ \\
\hline
\end{tabular}

30 minutos o hasta perder al grupo de vista, en este momento se continuó con el recorrido siguiendo las rutas establecidas. Esta metodología permite censar los estados de comportamiento de manera periódica en el grupo bajo diversas condiciones (Martin \& Bateson, 2007). Durante el escaneo se observaron partes distintas del grupo, para registrar distintos individuos. En grupos dispersos mayores de 50 individuos, se seleccionó un subgrupo para realizar los escaneos.

Análisis estadístico: Para el análisis se utilizaron todos los registros que contaban con la observación de los primeros 5 minutos de cada actividad y los escaneos siguientes. Para asociar los comportamientos de cada especie con la época de muestreo, se realizó una sumatoria de las frecuencias de presencia para cada comportamiento en cada avistamiento. Luego se agregaron las frecuencias por época seca y lluviosa y se analizó con una prueba de
Chi-cuadrado $(\chi 2)$ de independencia, similar a estudios que se han realizado con anterioridad en el país (Cubero-Pardo, 1998; May-Collado \& Morales-Ramírez, 2005). Debido a que los avistamientos poseían distintos números de escaneos y se realizaron en horas distintas, el cálculo de los valores esperados se corrigió según la proporción de escaneos observados por avistamiento en cada época.

\section{RESULTADOS}

Un total de 159 horas efectivas de muestreo fueron invertidas en 22 días de trabajo, entre los meses de mayo 2005 a abril 2006. Los recorridos mensuales cubrieron un área aproximada $174 \mathrm{~km}^{2}$. Durante los recorridos se registraron tres especies de cetáceos, en un total de 36 encuentros y 421 observaciones de comportamiento. El promedio general de encuentro por kilómetro recorrido fue de $0.073( \pm 0.079)$. En los meses de setiembre- octubre de 2005 
TABLA 2

Frecuencia de observaciones de los estados de comportamiento para cada especie de cetáceo por época del año, Pacífico norte de Costa Rica, 2015-2016.

TABLE 2

Frequency of observations of the behavioral states for each species by season, North Pacific of Costa Rica, 2015-2016.

\begin{tabular}{|c|c|c|c|c|c|c|}
\hline \multirow[t]{2}{*}{ Comportamiento } & \multicolumn{2}{|c|}{$\begin{array}{c}\text { Ballena jorobada } \\
\text { Megaptera novaeangliae }\end{array}$} & \multicolumn{2}{|c|}{$\begin{array}{l}\text { Delfín manchado } \\
\text { Stenella attenuata }\end{array}$} & \multicolumn{2}{|c|}{$\begin{array}{c}\text { Delfín nariz de botella } \\
\text { Tursiops truncatus }\end{array}$} \\
\hline & Lluviosa & Seca & Lluviosa & Seca & Lluviosa & Seca \\
\hline Forrajeo & 0 & 0 & 29 & 6 & 6 & 0 \\
\hline Desplazamiento-Forrajeo & 0 & 0 & 24 & 26 & 0 & 22 \\
\hline Alimentación & 0 & 0 & 13 & 0 & 0 & 0 \\
\hline Descanso & 0 & 0 & 5 & 0 & 0 & 0 \\
\hline Desplazamiento & 71 & 7 & 39 & 3 & 0 & 0 \\
\hline Socialización & 24 & 0 & 3 & 16 & 0 & 0 \\
\hline Madre-cría & 32 & 0 & 0 & 0 & 0 & 0 \\
\hline Buceo & 0 & 95 & 0 & 0 & 0 & 0 \\
\hline
\end{tabular}

y febrero-marzo de 2006, no hubo esfuerzo de muestreo debido a las malas condiciones ambientales. Debido a las pocas observaciones de T. truncatus, los análisis de comportamiento se realizaron solo para la ballenas jorobadas y delfines manchados (Tabla 2).

Los delfines manchados registraron la mayor variedad de comportamientos. La época lluviosa presentó mayor variedad de comportamientos que la época seca y de los cuatro comportamientos presentes, solo el forrajeo $\left(\mathrm{X}^{2}=\right.$ 29.42; g.1. $=1 ; \mathrm{P}<0.001)$ y el desplazamiento $\left(\mathrm{X}^{2}=38.33\right.$; g.l. $\left.=1 ; \mathrm{P}<0.001\right)$ aumentaron significativamente en esta época. De una manera menor a la esperada, el desplazamientoforrajeo $\left(\mathrm{X}^{2}=29.54\right.$; g.1. $\left.=1 ; \mathrm{P}<0.001\right)$ y el comportamiento social $\left(\mathrm{X}^{2}=6.83\right.$; g.1. $=1$; $\mathrm{P}<0.001)$ aumentó en la estación seca.

La ballena jorobada se observó en comportamientos de madre-cría durante las dos épocas para cada subpoblación. Al igual que con los delfines, la época lluviosa presentó una mayor variedad de comportamientos que la época seca. Los comportamientos predominantes fueron madre-cría, social y desplazamiento. Este último resultó mayor en la época lluviosa $\left(\mathrm{X}^{2}\right.$ $=78.16$; g.l. $=1 ; \mathrm{P}<0.001)$. Durante la época seca el $95 \%$ de los encuentros correspondieron al comportamiento de buceo.

\section{DISCUSIÓN}

Los resultados de este estudio concuerdan con estudios anteriores que demuestran que las aguas del Pacífico Norte de Costa Rica son un hábitat importante para los delfines manchados y ballenas jorobadas (Martínez-Fernández et al., 2011; May-Collado \& Forcada, 2012; MayCollado \& Morales-Ramírez, 2005). Hubo muchas limitantes ambientales que imposibilitaron aumentar el esfuerzo de muestreo y detallar los comportamientos de cada especie en cada época. Pero aun así, fue posible determinar que para los delfines manchados se presentó una estacionalidad en los comportamientos de forrajeo durante la época de lluvias, similar a lo reportado por May-Collado y MoralesRamírez (2005) en la misma zona de estudio. No fue el mismo caso con el comportamiento de socialización, que fue mayor en la época seca, quizá afectado por falta de datos a causa de las condiciones del clima.

$\mathrm{Si}$ bien los estudios de seguimiento y hábitos alimenticios sugieren que el delfín manchado en el Pacífico Tropical Oriental se alimenta principalmente de noche en las zonas epipelágicas y mesopelágicas (Baird et al., 2001; Scott \& Cattanach, 1998) este estudio no pudo contemplar los horarios nocturnos ni 
pelágicos, por lo hubo una limitación temporal y espacial para la colecta de los datos. Aun así, sí se considera que el ámbito de hogar de una especie se resume como la porción del área que esta utiliza para realizar actividades diarias (Burt 1943; Gubbins 2002), se puede reafirmar una vez más que la población del delfín manchado de la zona es residente, ya que esta especie utiliza la zona costera para realizar todos sus comportamientos a lo largo de todo el año. Sin embargo, aún queda por determinar patrones individuales de residencia en próximas investigaciones. La marcada presencia de comportamientos de forrajeo en la zona quizá se debe a que en el área durante la época seca se reporta un afloramiento producto del empuje de los vientos (Alfaro, 2014; Alfaro et al., 2012; Bianchi, 1991; Fiedler, Philbrick, \& Chavez, 1991), en donde hay mayor productividad y posiblemente mayor presencia de presas en la columna de agua para los delfines.

Para las ballenas jorobadas se han descrito tanto la presencia estacional como los movimientos migratorios desde los trópicos a las zonas templadas para alimentarse por dos poblaciones distintas (Calambokidis et al., 2001; Rasmussen et al., 2002; Rasmussen et al., 2007; Rasmussen et al., 2012; Steiger et al., 1991). Por su condición de especie altamente migratoria, hay comportamientos que tienden a ser estacionales, como el reproductivo. Es así como en este estudio solo se pudo comprobar la frecuencia de comportamiento en época reproductiva para las ballenas que llegan a la zona costera del área de estudio. Que los encuentros de madre-cría y de desplazamiento se hayan observado más en la época lluviosa durante la presencia de la población reproductiva del stock $G$, puede deberse a que las condiciones ambientales fueron más favorables para la identificación de ese comportamiento. Sin embargo, la proporción de estos encuentros (16.6\%) fue semejante al promedio de $17 \%$ reportado para los años 1996-2002 (Rasmussen et al., 2002) en la zona del Pacífico sur de Costa Rica. La dominancia de los comportamientos de madre-cría es de esperar en esos meses para esa población, ya que hay diferencia en el número de individuos de cada subpoblación de la especie. La población del DPS Centroamericano ronda los 500 a 600 individuos, mientras que la población reproductiva del stock $\mathrm{G}$ es cercana a los 6504 (95\% IC: entre 4270-907 individuos) (Bettridge et al., 2015).

Se confirma así que las aguas del Área de Conservación Guanacaste representan sitios de importancia para los cetáceos en el país. La identificación de comportamientos, como forrajeo en los delfines y sociales tipo madrecría en las ballenas, hacen que en la zona pueda existir un hábitat crítico para estas especies de cetáceos. Más aún cuando se trata de especies altamente migratorias como las ballenas jorobadas. Debido a que muchas especies de cetáceos se encuentran amenazadas en la actualidad (Bettridge et al., 2015; Schipper et al., 2008), es importante continuar con estudios de identificación y modelaje para poder predecir estos hábitat críticos, así como el modelaje de distribución de presas, para poder desarrollar estrategias de conservación apropiadas.

Declaración de ética: el autor declara que está de acuerdo con esta publicación; que no existe conflicto de interés de ningún tipo; y que ha cumplido con todos los requisitos y procedimientos éticos y legales pertinentes. Todas las fuentes de financiamiento se detallan plena y claramente en la sección de agradecimientos. El respectivo documento legal firmado se encuentra en los archivos de la revista.

\section{AGRADECIMIENTOS}

Se agradece la colaboración de Andrea Montero Cordero, David Palacios, Pablo Solís, Federico Pochet, Elena Echandi, Laura MayCollado, Manuel Spinola, Anibal Lara y Minor Lara. Al ICOMVIS, Fundación Keto, MICIT, CONICIT, Project A.W.A.R.E, Rufford Foundation, Cascadia Research Collective, U.S. Fish and Wildlife Service y Cetacean Society International. Se agradece los valiosos aportes de los revisores Laura May-Collado y Luis Fonseca al manuscrito, así como revisores anónimos. 


\section{RESUMEN}

Introducción: Tres especies de cetáceos, el delfín manchado pantropical (Stenella attenuata attenuata), el delfín nariz de botella (Tursiops truncatus) y la ballena jorobada (Megaptera novaeangliae) son un componente importante de la fauna marina del Área de Conservación Guanacaste. Sin embargo, la información biológica básica sobre el comportamiento y la presencia de estas especies sigue siendo escasa.

Objetivo: El objetivo de este estudio es determinar el comportamiento diurno de los cetáceos en el sector marino de la ACG y en sus áreas adyacentes.

Métodos: Se muestreó el Pacífico norte de Costa Rica desde Bahía Salinas hasta las Islas Murciélago, incluyendo el Golfo de Santa Elena, entre mayo de 2005 y mayo de 2006. Se utilizó el método de escaneo de comportamiento para identificar el comportamiento general de los grupos.

Resultados: Se registraron encuentros para tres especies en un total de 36 avistamientos, pero solo con un tiempo considerable para dos especies, S. attenuata y M. novaeangliae. Los delfines manchados exhibieron una amplia variedad de comportamientos en la temporada de lluvias. La búsqueda de alimento $\left(\mathrm{X}^{2}=29.42 ;\right.$ g.1. $\left.=1 ; \mathrm{P}<0.001\right)$ y los viajes $\left(\mathrm{X}^{2}=38.33\right.$; g.1. $\left.=1 ; \mathrm{P}<0.001\right)$ aumentaron significativamente en la época de lluvias. Los comportamientos predominantes para la ballena jorobada fueron la asociación madre-cría, social y de viaje; este último fue mayor en la época de lluvias $\left(\mathrm{X}^{2}=78.16\right.$; g.1. $=1$; $\mathrm{P}<0.001)$.

Conclusiones: En la zona, los cetáceos presentaron comportamientos como forrajeo y madre-cría, lo que significa que el área puede clasificarse como un hábitat crítico para estas especies de cetáceos, de mayor importancia para especies altamente migratorias como las ballenas jorobadas.

Palabras clave: delfines; ballenas jorobadas; Stenella attenuata; Megaptera novaeangliae; comportamiento de cetáceos; conservación marina.

\section{REFERENCIAS}

Acevedo, A., \& Smultea, M. A. (1995). First records of humpback whales including calves at Golfo Dulce and Isla del Coco, Costa Rica, suggesting geographical overlap of Northern and Southern Hemisphere populations. Marine Mammal Science, 11(4), 554-60. https://doi.org/10.1111/j.1748-7692.1995.tb00677.x

Acevedo, J., Aguayo-Lobo, A., Allen, J., Botero-Acosta, N., Capella, J., Castro, C., ... \& Stevick, P. T. (2017). Migratory preferences of Humpback Whales between feeding and breeding grounds in the Eastern South Pacific. Marine Mammal Science, 33(4), 1035-1052. https://doi.org/10.1111/mms.12423
Alfaro, E. J. (2014). Caracterización del 'Veranillo' en dos cuencas de la Vertiente del Pacífico de Costa Rica, América Central. Revista de Biología Tropical, 62(Suplemento 4), S1-S15. https://doi.org/10.15517/ rbt.v62i4.20010.

Alfaro, E. J., \& Cortés, J. (2012). Atmospheric forcing of cool and subsurface water events in Bahía Culebra, Gulf of Papagayo, Costa Rica. Revista de Biología Tropcical, 60(Supplement 2), S173-S186. https://doi. org/10.15517/RBT.V60I2.20001

Alfaro, E. J., \& Cortés, J. (2021). Forcing of cool and warm subsurface water events in Bahía Salinas, Costa Rica. Revista de Biología Tropical, 69(Supplement 2), S127-S141.

Alfaro, E. J., Cortés, J., Alvarado, J. J., Jiménez, C., León, A., Sánchez-Noguera, C., Nivia-Ruiz, J., \& Ruiz, E. (2012). Clima y temperatura sub-superficial del mar en Bahía Culebra, Golfo de Papagayo, Costa Rica. Revista de Biología Tropical, 60(Supplement 2), S159-S171. https://doi.org/10.15517/rbt.v60i2.20000

Altmann, J. (1974). Observational study of behavior: bampling methods. Behaviour, 49(3-4), 227-66. https:// doi.org/10.1163/156853974X00534

Baird, R. W., \& Dill, L. M. (1995). Occurrence and behaviour of transient Killer Whales: seasonal and pod-specific variability, foraging behaviour, and prey handling. Canadian Journal of Zoology, 73(7), 13001311. https://doi.org/10.1139/z95-154

Baird, R. W., Ligon, A. D., Hooker, S. K., \& Gorgone, A. M. (2001). Subsurface and nighttime behavior of Pantropical Spotted Dolphins in Hawaii. Canadian Journal of Zoology, 79, 988-96. https://doi. org/10.1139/z01-070

Bassey, F. G. (2010). Evaluación ecológica de los arrecifes y comunidades coralinas de las Islas Murciélago y sección norte de la Península de Santa Elena, Pacífico de Costa Rica (Tesis Maestría). Universidad Nacional de Costa Rica, Puntarenas, Costa Rica.

Bettridge, S. C., Baker, S., Barlow, J., Clapham, P. J., Ford, M., Gouveia, D., ...\& Wade, P. R. (2015). Status review of the Humpback Whale (Megaptera novaeangliae) under the Endangered Species Act. Department of Commerce, NOAA Technical Memorandum NMFS NOAA-TMNMFS-SWFSC-540. National Oceanic and Atmospheric Administration, National Marine Fisheries Service, Southwest Fisheries Science Center, La Jolla.

Bianchi, G. (1991). Demersal assemblages of the continental shelf and slope edge between the Gulf of Tehuantepec (Mexico) and the Gulf of Papagayo (Costa Rica). Marine Ecology Progress Series, 73(2-3), 121-40. https://doi.org/10.3354/meps073121 
Burt, W. H. (1943). Territoriality and home range concepts as applied to mammals. Journal of Mammalogy, 24(3), 346-352.

Calambokidis, J., Steiger, G. H., Rasmussen, K., Urbán, J. R., Balcomb, K. C., Ladrón De Guevara, P. P., ... Darling, J. D. (2000). Migratory destinations of Humpback Whales that feed off California, Oregon and Washington. Marine Ecology Progress Series, 192, 295-304. https://doi.org/10.3354/meps192295

Calambokidis, J., Steiger, G. H., Straley, J. M., Herman, L. M., Cerchio, S., Salden, D. R.,... \& Quinn, T. J. (2001). Movements and population structure of Humpback Whales in the North Pacific. Marine Mammal Science, 17(4), 769-94. https://doi. org/10.1111/j.1748-7692.2001.tb01298.x

Cortés, J. (1996). Comunidades coralinas y arrecifes del Área de Conservación Guanacaste, Costa Rica Revista de Biología Tropical, 44(3), 623-625.

Cortés, J. (2017). Marine biodiversity baseline for Área de Conservación Guanacaste, Costa Rica: published records. ZooKeys, 652, 129-179. https://doi. org/10.3897/zookeys.652.10427

Cubero-Pardo, P. (1998). Distribución y patrones de actividad del bufeo (Tursiops truncatus) y el delfín manchado (Stenella attenuata) en el Golfo Dulce, en relación con variables ambientales (Tesis de maestría). Universidad de Costa Rica, San José, Costa Rica.

Fiedler, P. C., Philbrick, V., \& Chavez, F. P. (1991). Oceanic upwelling and productivity in the Eastern Tropical Pacific. Limnology and Oceanography, 36(8), 18341850. https://doi.org/10.4319/lo.1991.36.8.1834

Fonseca, L. G., Murillo, G. A., Guadamúz, L., Spínola, R. M., \& Valverde, R. A. (2009). Downward but stable trend in the abundance of arribada olive ridley sea turtles (Lepidochelys olivacea) at Nancite Beach, Costa Rica (1971-2007). Chelonian Conservation and Biology, 8(1), 19-27. https://doi.org/10.2744/ ccb-0739.1

Fonseca, L. G., Santidrián-Tomillo, P., Villachica, W. N., Quirós, W. M., Pesquero, M., Heidemeyer, M.,... \& Valverde, R. A. (2019). Discovery of a major East Pacific green turtle (Chelonia mydas) nesting population in Northwest Costa Rica. Chelonian Conservation and Biology, 17(2), 169. https://doi.org/10.2744/ ccb-1264.1

Gubbins, C. (2002). Use of home ranges by resident bottlenose dolphins (Tursiops truncatus) in a South Carolina estuary.JournalofMammalogy, 83(1),178-187.https:// doi.org/10.1644/1545-1542(2002)083<0178:uohrbr> 2.0.co;2

Loría-Naranjo, M., Samper-Villarreal, J., \& Cortés, J. (2014). Structural complexity and species composition of Potrero Grande and Santa Elena mangrove forests in Santa Rosa National Park, North Pacific of Costa Rica. Revista de Biología Tropical, 62(Supplement 4), S33-S41. https://doi.org/10.15517/rbt. v62i4. 20030

Mann, J. (2000). Unraveling the dynamics of social life: long-term studies and observation methods. In: J. Mann, R. C., Connonr, P. L. Tyack, \& H. Whitehead (Eds.), Cetacean societies: field studies of dolphins and whales (pp. 45-64). Chicago: University of Chicago Press.

Martin, P., \& Bateson, P. (2007). Measuring behaviour: an introdution guide. 3rd Ed. Cambridge: Cambridge University Press.

Martínez-Fernández, D., Montero-Cordero, A., \& MayCollado, L. (2011). Cetáceos de las aguas costeras del Pacífico Norte y Sur de Costa Rica. Revista de Biología Tropical, 59(1), 283-90. https://doi.org/10.15517/ rbt.v59i1.3197

Martínez-Fernández, D., Montero-Cordero, A., \& PalaciosAlfaro, D. (2014). Áreas de congregación de cetáceos en el Pacífico Norte de Costa Rica: recomendaciones para la gestión del recurso. Revista de Biologia Tropical, 62(Suplemento 4), S99-S108. https://doi. org/10.15517/RBT.V62I4.20035

May-Collado, L., \& Forcada, J. (2012). Small-scale estimation of relative abundance for the coastal spotted dolphins (Stenella attenuata) in Costa Rica: the effect of habitat and seasonality. Revista de Biología Tropical, 60(Supplemement 2), S133-S142. https://doi. org/10.15517/rbt.v60i2.19997

May-Collado, L., Gerrodette, T., Calambokidis, J., Rasmussen, K., \& Sereg, I. (2005). Patterns of cetacean sighting distribution in the Pacific Exclusive Economic Zone of Costa Rica based on data collected from 1979-2001. Revista de Biología Tropical, 53(1-2), 249-263. https://doi.org/10.15517/rbt.v53i1-2.14556

May-Collado, L., \& Morales-Ramírez, A. (2005). Occurrence and behavioral patterns of the spotted coastal dolphin Stenella attenuata (Cetacea: Delphinidae) in the Gulf of Papagayo, Costa Rica. Revista de Biología Tropical, 53(1-2), 265-276. https://doi. org/10.15517/rbt.v53i1-2.14559

Palacios-Alfaro, J. D., Martínez-Fernández, D., Sánchez, C., \& Venegas-Li, R. (2009). Distribution and behavior of humpback whale (Megaptera novaeangliae Borowski, 1781) (Breeding Stock G), in Southern Pacific of Costa Rica. Journal of Cetacean Research Management, 12(1), 1-8.

Rasmussen, K., Calambokidis, J., \& Steiger, G. H. (2002). Humpback whales and other marine mammals off Costa Rica and surrounding waters, 1996-2002. Report of the Oceanic Society 2002 field season in cooperation with Elderhostel volunteers. San Francisco, California: Oceanic Society Expeditions. 
Rasmussen, K., Calambokidis, J., \& Steiger, G. H. (2012). Distribution and migratory destinations of Humpback Whales off the Pacific Coast of Central America during the boreal winters of 1996-2003. Marine Mammal Science, 28(3), E267-E279. https://doi. org/10.1111/j.1748-7692.2011.00529.x

Rasmussen, K., Palacios, D. M., Calambokidis, J., Saborío, M. T., Dalla Rosa, L., Secchi,... \& Stone, G. S. (2007). Southern Hemisphere humpback whales wintering off Central America: insights from water temperature into the longest mammalian migration. Biology Letters, 3(3), 302-305. https://doi. org/10.1098/rsbl.2007.0067

Schipper, J., Chanson, J. S., Chiozza, F., Cox, N. A., Hoffmann, M., Katariya, V., ... \& Young, B. E. (2008). The status of the world's land and marine mammals: diversity, threat, and knowledge. Science, 322(5899), 225-230. https://doi.org/10.1126/science.1165115

Scott, M. D., \& Cattanach, K. L. (1998). Diel patterns in aggregations of pelagic dolphins and tunas in the Eastern Pacific. Marine Mammal Science, 14(3),
401-422. https://doi.org/10.1111/j.1748-7692.1998. tb00735.x

SINAC. (2008). GRUAS II: Propuesta de ordenamiento territorial para la conservación de la biodiversidad de Costa Rica. San José, Costa Rica: Ministerio de Ambiente y Energía.

Steiger, G. H., Calambokidis, J., Sears, R., Balcomb, K. C., \& Cubbage, J. C. (1991). Movement of Humpback Whales between California and Costa Rica. Marine Mammal Science, 7(3), 306-310. https://doi. org/10.1111/j.1748-7692.1991.tb00105.x

Waylen, P. R., Caviedes, C. N., \& Quesada, M. E. (1996). Interannual variability of monthly precipitation in Costa Rica. Journal of Climate, 9(10), 2606-2613. doi: 10.1175/1520-0442(1996)009<2606:IVOMPI> 2.0. $\mathrm{CO} ; 2$

Zamora-Trejos, P., \& Cortés, J. (2009). Los manglares de Costa Rica: el Pacífico Norte. Revista de Biología Tropical, 57(3), 473-488. https://doi.org/10.15517/ rbt.v57i3.5469 